\title{
SYSTEMS OF LINEAR DIFFERENCE EQUATIONS AND EXPANSIONS IN SERIES OF EXPONENTIAL FUNCTIONS*
}

\author{
BY \\ R. D. CARMICHAEL
}

Introduction. The principal purpose of the first part of this paper is to prove ( $\$ 1.9)$ that the system (1.1) of linear non-homogeneous generalized difference equations has solutions $g_{k}(x), k=1,2, \cdots, n$, which are integral functions provided that the independent terms $\phi_{\nu}(x)$ are themselves integral functions and provided that the system has a certain non-singular character defined in $\$ 1.3$. In case the $\phi_{\nu}(x)$ are further restricted to be of exponential type ( $\$ 1.5)$ then solutions of exponential type exist ( $\$ 1.6)$ and indeed solutions of exponential type at most equal to $q$ (called principal solutions) in case no $\phi_{\nu}(x)$ is of higher type than $q$ and at least one of them is of precisely this type. A useful symbolic notation $(\$ 1.2)$ is effective in carrying out the argument.

In the second part of the paper we apply the results of the first part to the rather remarkable problem of the simultaneous expansion of $n$ integral functions in composite power series, a problem which we have not seen treated elsewhere.

The third part of the paper is devoted to the theory of a class of remarkable expansions in series of exponential functions, generalizing the theory of Fourier series. Whereas the basic region of convergence of Fourier series is a segment of a straight line, these new series, apart from certain particular cases, have certain polygons in the complex plane as their basic regions of convergence. The vertices of these polygons play the rôle of the end points of the segments in the case of Fourier series, while the remaining points of the polygon play the rôle of interior points of the segments. Several extensions of the theory are briefly indicated (\$3.4) and an application is made ( $\$ 3.5)$ to the expansion of Bernoulli polynomials of higher order in series of exponential functions.

\section{ON A SYSTEM OF LINEAR DIFFERENCE EQUATIONS WITH CONSTANT COEFFICIENTS}

1.1. Formulation of the problem. We consider the problem of solving the system

* Presented to the Society, August 31, 1932; received by the editors June 27, 1932. 


$$
\sum_{j=1}^{n} c_{\nu} g_{j}\left(x+a_{v j}\right)=\phi_{\nu}(x) \quad(\nu=1,2, \cdots, n)
$$

of functional equations (generalized linear difference equations with constant coefficients), where the functions $\phi_{\nu}(x), \nu=1,2, \cdots, n$, are $n$ given integral functions and the $n$ functions $g_{j}(x), j=1,2, \cdots, n$, are to be determined subject to the requirement that they shall be integral functions. In this system the coefficients $c_{v j}$ and the additive terms $a_{v j}$ in the arguments are given constants; in $\$ 1.3$ we shall subject these constants to a certain negative condition in order to avoid exceptional cases in the theory of the system.

We- shall sometimes subject the $\phi_{\nu}(x)$ to additional restrictions and in such cases we shall put like further restrictions upon the solutions $g_{j}(x)$, thus obtaining what may be called principal solutions of the given system.

The theory of system (1.1) contains that of the single equation

$$
\sum_{k=1}^{\mu} \gamma_{k} F\left(x+\alpha_{k}\right)=G(x)
$$

where $\alpha_{1}, \alpha_{2}, \cdots, \alpha_{\mu}$ are different constants, $\gamma_{1}, \gamma_{2}, \cdots, \gamma_{\mu}$ are constants different from $0, G(x)$ is an integral function, and $F(x)$ is to be determined as an integral function. To see this it is sufficient to write $g_{k}(x)=F\left(x+\alpha_{k}\right)$ and to form the system

$$
g_{1}\left(x-\alpha_{1}\right)-g_{k}\left(x-\alpha_{k}\right)=0, \quad k=2,3, \cdots, \mu, \quad \sum_{k=1}^{\mu} \gamma_{k} g_{k}(x)=G(x) .
$$

This system is of the form (1.1). From a solution of this system we have a solution of equation (1.2); and vice versa.

In a similar way one may reduce the problem of solving a system generalizing (1.1) and (1.2) at the same time to the problem of solving a system of the same form as (1.1).

Special cases of the problem here set have been treated by various authors.*

1.2. Introduction of symbolic operators. We define the symbolic operator $E(a)$ by the relation

$$
E(a) \cdot f(x) \equiv f(x+a) .
$$

A linear homogeneous combination of such operators will have the meaning indicated by the relation

* See, for instance, C. Guichard, Annales de l'Ecole Normale Supérieure, (3), vol. 4 (1887), pp. 361-380; A. Hurwitz, Acta Mathematica, vol. 20 (1897), pp. 285-312; S. Pincherle, Ibid., vol. 48 (1926), pp. 279-304 (first published in 1888); R. D. Carmichael, American Journal of Mathematics, vol. 35. (1913), pp. 163-182; E. Hilb, Mathematische Annalen, vol. 85 (1922), pp. 89-98. 


$$
\left\{\sum_{k=1}^{\mu} \gamma_{k} E\left(a_{k}\right)\right\} \cdot f(x) \equiv \sum_{k=1}^{\mu} \gamma_{k} f\left(x+a_{k}\right)
$$

This will serve, in particular, to define the sum and the difference of two operators of the form $\alpha E(a)$ and $\beta E(b)$. The product of these two operators is defined by the formula

$$
\alpha E(a) \cdot \beta E(b)=\alpha \beta E(a+b) .
$$

These definitions serve to give a unique meaning to any polynomial combination of operators of the form $E\left(a_{k}\right)$, the coefficients being constants. Such a polynomial in operators $E$ may be written as a linear function of suitably defined operators $E$, as one sees by aid of (1.5). In particular, one may define such an operator by means of a symbolic determinant of the form

$$
\Delta \equiv\left|c_{\nu j} E\left(a_{\nu j}\right)\right|
$$

whose element in $\nu$ th row and $j$ th column is $c_{v j} E\left(a_{v j}\right)$, this being (by definition) the symbolic operator obtained by expanding the determinant formally as if its elements were ordinary algebraic quantities. The expanded determinant may be written as a linear homogeneous function of suitable operators $E$ with constant coefficients.

Any polynomial combination of such operators $E$ will be said to have the value zero when and only when the result of operating with it upon an arbitrary integral function gives the value zero identically. It is easily shown that such a polynomial in operators $E$ is zero if and only if the function $e^{x t}$ of $x$ is reduced to zero for all $t$ when operated upon by the named operator.

1.3. Symbolic form of (1.1); restriction on the system. Employing the symbolic operators introduced in $\$ 1.2$ we write system (1.1) in the form

$$
\sum_{j=1}^{n} c_{\nu j} E\left(a_{\nu j}\right) \cdot g_{j}(x)=\phi_{\nu}(x) \quad(\nu=1,2, \cdots, n) .
$$

The determinant $\Delta$ in (1.6) will be called the symbolic determinant of system (1.7). This determinant will be called singular when it has the value zero; otherwise it will be called non-singular.

We shall treat system (1.1) or (1.7) only in the case when its determinant is non-singular. In that case we shall say that the system is non-singular. For the treatment of the excluded exceptional case the methods required are quite different from those here employed.

We shall use (without further definition) the terms customarily employed in the theory of determinants. 
1.4. Separation of variables. Let $A_{v j}$ denote the cofactor of the element in the $\nu$ th row and $j$ th column of $\Delta$. Then $A_{v j}$ is a polynomial in operators $E$ with constant coefficients. Moreover, we have

$$
\sum_{j=1}^{n} c_{\nu j} E\left(a_{v j}\right) A_{v j}=\delta_{v k} \Delta, \quad \sum_{\nu=1}^{n} c_{v j} E\left(a_{\nu j}\right) A_{\nu k}=\delta_{j k} \Delta,
$$

where $\delta_{\nu k}$ is 1 or 0 according as $\nu=k$ or $\nu \neq k$.

Multiplying the $\nu$ th equation in (1.7) by the operator $A_{\nu k}$, summing as to $\nu$ from 1 to $n$, interchanging the order of summations in the first member of the resulting equation and simplifying by aid of the second equation in (1.8), we have

$$
\Delta g_{k}(x)=\sum_{\nu=1}^{n} A_{\nu k} \phi_{\nu}(x) \quad(k=1,2, \cdots, n) .
$$

In these $n$ equations the unknown functions $g_{k}(x)$ appear singly. Any solution of (1.7) must satisfy (1.9).

The operator $\Delta$ may be written in the form

$$
\Delta \equiv \sum_{k=0}^{\sigma} c_{k} E\left(a_{k}\right)
$$

where the $c_{k}$ are constants different from zero and the $a_{k}$ are different constants (this form being surely possible since $\Delta$ is non-singular). The value of $\sigma$ depends on $n$ and the constants $c_{v j}$ and $a_{v j}$; it is never greater than $n !-1$.

If $\sigma=0$ the required inverse operator $\Delta^{-1}$ is $E\left(-a_{0}\right) / c_{0}$. In this case a complete solution is readily obtained and the problem is trivial.

If $\sigma>0$, as we shall henceforth assume it to be, we may write each equation (1.9) in the form

$$
c_{0} g\left(x+a_{0}\right)+c_{1} g\left(x+a_{1}\right)+\cdots+c_{\sigma} g\left(x+a_{\sigma}\right)=\phi(x),
$$

where $\phi(x)$ is a given function and $g(x)$ is to be determined. From the solution of such an equation as this we shall pass to the solution of system (1.7).

We shall find it convenient to employ the function $h(t)$ defined by the equation

$$
h(t)=e^{-x t} \cdot \Delta \cdot e^{x t} .
$$

Since the given system is non-singular it follows that $h(t)$ is not identically zero.

We have seen that every solution of (1.7) is a solution of (1.9). But the converse does not hold, as we shall now show. If $g_{k}(x), k=1,2, \cdots, n$, is any solution of (1.9) then its general solution is $g_{k}(x)+p_{k}(x), k=1,2, \cdots, n$, 
where the functions $p_{k}(x)$ are arbitrary functions satisfying the equation $\Delta p_{k}(x)=0$. Now consider the system

$$
\begin{aligned}
g_{1}(x+1)+g_{2}(x)+g_{3}(x+2)= & \phi_{1}(x), g_{1}(x+2)+g_{2}(x)+g_{3}(x+1)=\phi_{2}(x), \\
& g_{3}(x)=\phi_{3}(x) .
\end{aligned}
$$

Here we have $\Delta=E(1)-E(2) \neq 0$, whereas the function $g_{3}(x)$ is uniquely determined by the last equation in the system.

From this example it follows that it is necessary to obtain an appropriate solution of (1.9) in order to have a solution of (1.7). The problem falls naturally into two cases; the following section prepares the way for this separation of cases.

1.5. Functions of exponential type. If $f(x)$ is an analytic function which is regular at $x_{0}$ and $x_{1}$, then it is easily shown that

$$
\limsup _{\nu=\infty}\left|f^{(\nu)}\left(x_{0}\right)\right|^{1 / \nu}=\limsup _{\nu=\infty}\left|f^{(\nu)}\left(x_{1}\right)\right|^{1 / \nu},
$$

where the superscripts denote derivatives with respect to $x$. If these superior limits have the finite value $q(q \geqq 0)$ then $f(x)$ is an integral function; in such a case we shall say that $f(x)$ is of exponential type $q$, this terminology being justified by the following theorem, ${ }^{*}$ stated here without proof:

THEOREM 1.1. A necessary and sufficient condition that the function $f(x)$ shall be of exponential type $q$ is (1) that numbers $\tau$ shall exist for which it is true that for every positive number $\epsilon$ there exists a quantity $M$, depending on $\epsilon$ and $\tau$ in general but independent of $x$, such that for all (finite) values of $x$, we have

$$
|f(x)|<M e^{(r+\epsilon)|x|}
$$

and (2) that $q$ shall be the least possible value for such numbers $\tau$. Moreover, when $f(x)$ is of exponential type $q$, we have

$$
\left|f^{(\nu)}(x)\right|<M(q+\epsilon)^{\nu} e^{(q+\epsilon)|x|} \quad(\nu=0,1,2, \cdots),
$$

where $M$ is independent of $x$ and $\nu$.

1.6. Case when the $\phi_{\nu}(x)$ are of exponential type. We first carry out the solution of (1.7) for the case when the known functions $\phi_{\nu}(x)$ are of exponential type not exceeding $q$. Taking their power series expansions in the form

$$
\phi_{\nu}(x)=\sum_{k=0}^{\infty} s_{\nu k} x^{k} / k ! \quad(\nu=1,2, \cdots, n),
$$

* For a proof of this theorem and for further properties of functions of exponential type $q$, together with references to the literature, see a forthcoming paper of mine in Annals of Mathematics. 
we introduce the functions $\psi_{v}(t)$ by means of the expansions

$$
\psi_{\nu}(t)=\frac{s_{\nu 0}}{t}+\frac{s_{\nu 1}}{t^{2}}+\frac{s_{\nu 2}}{t^{3}}+\cdots \quad(\nu=1,2, \cdots, n) .
$$

Then the series in (1.14) all converge if $|t|>q$. Let $r$ be a positive number exceeding $q$ such that the circle $C_{r}$ of radius $r$ about 0 as a center passes through no zero of the function $h(t)$ defined in (1.12). (This negative condition on $r$ is first needed in the next paragraph.) Then we have

$$
\phi_{\nu}(x)=\frac{1}{2 \pi i} \int_{C_{r}} e^{x t} \psi_{\nu}(t) d t \quad(\nu=1,2, \cdots, n),
$$

as one sees by using expansions (1.14) and integrating term by term in (1.15).

We employ the operator $A_{v j}$ with the meaning given in $\$ 1.4$. By $A_{v j} e^{x t}$ we mean the result of operating with $A_{v j}$ on $e^{x t}$ considered as a function of $x$. Now write

$$
g_{j}(x)=\frac{1}{2 \pi i} \int_{c_{r}} \sum_{k=1}^{n}\left(A_{k j} e^{x t}\right) \psi_{k}(t) \frac{d t}{h(t)} \quad(j=1,2, \cdots, n),
$$

this being suggested by the problem of solving (1.9) by the method employed by Pincherle (loc. cit.) for a similar equation. Now substitute in the first member of (1.7) the functions $g_{i}(x)$ so defined and simplify by aid of (1.8) and (1.15); thus we have

$$
\begin{aligned}
\sum_{j=1}^{n} c_{v j} E\left(a_{v j}\right) \cdot g_{j}(x) & =\frac{1}{2 \pi i} \int_{C_{r}} \sum_{k=1}^{n}\left[\sum_{j=1}^{n} c_{\nu j} E\left(a_{v j}\right) A_{k j} \cdot e^{x t}\right] \psi_{k}(t) \frac{d t}{h(t)} \\
& =\frac{1}{2 \pi i} \int_{C_{r}} \sum_{k=1}^{n} \delta_{\nu k}\left(\Delta e^{x t}\right) \psi_{k}(t) \frac{d t}{h(t)} \\
& =\frac{1}{2 \pi i} \int_{C_{r}} e^{x t} \psi_{\nu}(t) d t=\phi_{v}(x) .
\end{aligned}
$$

Therefore the functions $g_{j}(x)$ defined by (1.16) afford a solution of (1.7).

Equation (1.16) may be written in the form

$$
g_{j}(x)=\frac{1}{2 \pi i} \int_{C_{r}} e^{x t} \sum_{k=1}^{n}\left[A_{j} e^{x t}\right]_{x=0} \psi_{k}(t) \frac{d t}{h(t)} .
$$

Thence it follows that a constant $M$ exists such that

$$
\left|g_{i}^{(\nu)}(0)\right|<M r \cdot r^{\nu} \text {. }
$$

Therefore $g_{j}(x)$ is an integral function of exponential type not exceeding $r$. 
Suppose next that $r$ is so chosen that there is no zero of $h(t)$ in the interior of the circular ring bounded by $C_{r}$ and the circle $|t|=q$. Then $g_{j}(x)$ remains unaltered as $r$ decreases towards $q$ remaining greater than $q$. Therefore, in this case, the function $g_{j}(x)$ is of exponential type not greater than $q$. Furthermore, if in this case at least one of the functions $\phi_{\nu}(x), \nu=1,2, \cdots, n$, is of exponential type $q$ (none being of higher type), then at least one of the functions $g_{j}(x), j=1,2, \cdots, n$, is of exponential type $q$ and none is of higher type.

If the functions $\phi_{\nu}(x)$ are of exponential type $q$ or less and at least one of them is of type $q$ then a solution of (1.7) will be called a principal solution if no function in it is of exponential type exceeding $q$. We have just shown the existence of such principal solutions. To determine all principal solutions we have to find all solutions, of exponential type not exceeding $q$, of the homogeneous system corresponding to (1.7). This problem is left for a later investigation.

The main result in this section may be stated as in the following theorem:

THEOREM 1.2. When the $\phi_{\nu}(x)$ are functions of exponential type not greater than $q$ and one at least of them is of type $q$, then the non-singular system (1.1) or (1.7) admits as a principal solution the functions $g_{j}(x)$ defined by (1.16) for $r=q+\epsilon$, where $\epsilon$ is a small positive quantity such that $h(t)$ has no zero in the ring bounded by $C_{r}$ and the circle $|t|=q$.

1.7. Lemmas concerning exponential sums. Equations (1.9) are of the form (1.11). Replacing $x$ in (1.11) by $x-a_{0}$ we have another equation of the same form in which $a_{0}=0$. Hence there is no loss of generality in taking $a_{0}=0$; and this we do. Then the function $h(t)$ in (1.12) has the form

$$
h(t)=c_{0}+c_{1} e^{a_{1} t}+c_{2} e^{a_{2} t}+\cdots+c_{\sigma} e^{a_{\sigma} t} .
$$

In preparation for the treatment of the case when the functions $\phi_{\nu}(x)$ are general integral functions we state certain lemmas concerning the function $h(t)$.

We shall first determine certain infinite regions in which $h(t)$ is free of zeros and in fact is bounded away from zero. Separating $t$ and the $a_{k}$ into real and imaginary parts we write

$$
t=u+i v, a_{k}=\alpha_{k}+i \beta_{k} \quad(k=1,2, \cdots, \sigma) .
$$

Let $l_{\lambda \mu}$ denote the line

$$
l_{\lambda_{\mu}}: \quad R\left(a_{\lambda} t\right)=R\left(a_{\mu} t\right), \lambda \neq \mu,
$$

where $R(z)$ is the real part of $z$. Then $l_{\lambda \mu}$ and $l_{\mu \lambda}$ denote the same line. Moreover $l_{\lambda \mu}$ and $l_{\rho \tau}$ coincide if $a_{\lambda}-a_{\mu}=c\left(a_{\rho}-a_{\tau}\right)$ where $c$ is a real number; otherwise they do not coincide. Let $s$ denote the number of distinct lines in the set $l_{\lambda \mu}$. 
Since each of these $s$ lines passes through zero they divide the plane into $2 s$ sectors such that no point of any one of these lines is in the interior of any such sector.

Let $S_{1}$ be any one of these $2 s$ sectors and let $t_{1}$ be any given interior point of $S_{1}$. Then no two of the quantities $R\left(a_{k} t_{1}\right), k=0,1, \cdots, \sigma$, are equal. Let them be arranged in order of descending magnitude, thus:

$$
R\left(a_{k_{0}} t_{1}\right)>R\left(a_{k_{1}} t_{1}\right)>\cdots>R\left(a_{k_{\sigma}} t_{1}\right) .
$$

Then if $t_{1}$ varies continuously over the interior of $S_{1}$ this continued inequality will be preserved, since each member varies continuously and no two become equal for an interior point of $S_{1}$. One and just one term of this continued inequality is zero for an interior point $t_{1}$ of $S_{1}$. Hence the first term is not negative.

In the sector $S_{1}$ take a point $P$ which is at a distance $\delta$ from each of the bounding rays of the sector, where $\delta$ is a positive quantity whose value is to be assigned later. From $P$ draw rays to infinity in $S_{1}$ and parallel to the bounding rays of $S_{1}$, thus forming a new sector $S$ interior to $S_{1}$.

Let $(u, v)$ be any point in $S$. Then the distance from $(u, v)$ to the line $l_{k_{0} k_{1}}$ is the positive quantity

$$
\frac{\left(\alpha_{k_{0}}-\alpha_{k_{1}}\right) u-\left(\beta_{k_{0}}-\beta_{k_{1}}\right) v}{\left\{\left(\alpha_{k_{0}}-\alpha_{k_{1}}\right)^{2}+\left(\beta_{k_{0}}-\beta_{k_{1}}\right)^{2}\right\}^{1 / 2}} .
$$

But this distance is not less than $\delta$, whether $l_{k_{0} k_{1}}$ is or is not a bounding line of $S_{1}$. Therefore if $t$ denotes the point $(u, v)$ we have

$$
\begin{aligned}
R\left(a_{k_{0}} t\right)-R\left(a_{k_{1}} t\right)=\left(\alpha_{k_{0}}-\alpha_{k_{1}}\right) u-\left(\beta_{k_{0}}-\beta_{k_{1}}\right) v \\
\\
\geqq \delta\left\{\left(\alpha_{k_{0}}-\alpha_{k_{1}}\right)^{2}+\left(\beta_{k_{0}}-\beta_{k_{1}}\right)^{2}\right\}^{1 / 2} .
\end{aligned}
$$

Now let $\eta$ be a fixed quantity such that

$\left|c_{\tau}\right|\left(1+e^{-\eta}\right)-\left(\left|c_{0}\right|+\left|c_{1}\right|+\cdots+\left|c_{\sigma}\right|\right) e^{-\eta}>0 \quad(\tau=0,1, \cdots, \sigma)$.

Let $m$ be the least value attained by the left member as $\tau$ varies over the set $0,1, \cdots, \sigma$. Determine $\delta$ so that

$$
\delta\left\{\left(\alpha_{\lambda}-\alpha_{\mu}\right)^{2}+\left(\beta_{\lambda}-\beta_{\mu}\right)^{2}\right\}^{1 / 2} \geqq \eta, \lambda \neq \mu,
$$

for every pair of different numbers $\lambda$ and $\mu$ from the set $0,1, \cdots, \sigma$. Then $R\left(a_{k_{1}} t\right)-R\left(a_{k_{0}} t\right) \leqq-\eta$ for all $t$ in $S$. Hence $R\left(a_{k} t\right)-R\left(a_{k_{0}} t\right) \leqq-\eta$ for all $t$ in $S$ and for all $k$ in the set $0,1, \cdots, \sigma$ except $k=k_{0}$.

From these inequalities and the fact that $R\left(a_{k_{0}} t\right) \geqq 0$ in $S$ it follows readily that for all $t$ in $S$ we have

$$
|h(t)| \geqq m>0
$$


This inequality is independent of the particular sector $S$; hence it holds for all sectors $S$ formed (in the way indicated) by aid of a $\delta$ satisfying the named condition. We therefore have the following lemma:

Lemma 1.1. In the sectors $S$ formed as indicated the function $h(t)$ satisfies inequality (1.18).

When the sectors $S$ are cut out of the plane there is left a sort of infinite star in which lie all the zeros of $h(t)$ and in fact all the points $t$ for which $|h(t)|<m$. Thus we see that $h(t)$ is bounded away from zero in the distant part of the plane except possibly for certain regions in the star-arms remaining after removing the sectors $S$. We next consider the problem of bounding $h(t)$ away from zero in certain parts of these star-arms.

By a rotation of the $t$-plane, obtained by replacing $t$ by $e^{i \theta} t$ where $\theta$ is real, any particular bounding ray of any sector $S_{1}$ may be transformed to the positive part of the $u$-axis. Since this transformation leaves invariant the sort of result we are to establish we may (and we shall) temporarily suppose that this transformation has already been carried out; for convenience we retain the original notation. The star-arm to be considered will then lie along the positive real axis; we denote it by $A$. Then the real axis is a line $h_{\lambda \mu}$, and we have $\alpha_{\lambda}=\alpha_{\mu}$ while $\beta_{\lambda} \neq \beta_{\mu}$.

The maximum $\alpha_{k}$ is positive or zero, since $\alpha_{0}=0$. If the maximum value $\alpha$ of the $\alpha_{k}$ is the value of just one of them, then as $t$ becomes infinite in $A$, the function $|h(t)|$ becomes infinite or approaches a finite limit different from zero according as $\alpha$ is positive or zero. In this case $h(t)$ is bounded away from zero in the distant part of the star-arm.

In what remains we may therefore suppose that the maximum value $\alpha$ of the $\alpha_{k}$ is the value of two or more of them. Now in the star-arm $A$ we have

$$
\left|e^{-\alpha t} h(t)\right| \leqq|h(t)|,
$$

the sign of equality holding when and only when $\alpha=0$. But $e^{i \beta_{k} t}$ and its reciprocal are bounded in absolute value in $A$. It follows therefore that it is sufficient to treat only the special case in which $\alpha=0$, as may be seen by replacing $h(t)$ by a suitable $e^{-\left(\alpha+i \beta_{k}\right) t} h(t)$. Therefore we take $\alpha=0$. We temporarily choose the notation so that the values of $k$ for which $\alpha_{k}=0$ are $k=0,1, \cdots, \gamma-1$.

Write

$$
h_{1}(t)=\sum_{k=0}^{\gamma-1} c_{k} e^{\beta_{k}(-v+i u)} .
$$

Then $h(t)-h_{1}(t)$ approaches zero as $t$ becomes infinite in $A$. It is therefore 
sufficient to our purpose to determine suitable parts of $A$ in which $h(t)$ is different from zero and $h_{1}(t)$ is bounded away from zero.

For this investigation we need the following classical lemma which we state without proof:

LEMMA 1.2. If $b_{1}, b_{2}, \cdots, b_{\nu}$ is any set of real numbers, all different from zero, and if $\delta$ is any preassigned positive number, then there is an infinitude of positive integers $m$ such that, for each such $m$, integers $k_{1}, k_{2}, \cdots, k_{\nu}$ exist such that

$$
\left|k_{j} b_{j}+m\right| \leqq \delta \quad(j=1,2, \cdots, \nu) .
$$

If all such positive integers $m$ are denoted by the symbols $m_{1}, m_{2}, \cdots$, with $m_{j}<m_{j+1}, j=1,2, \cdots$, then among the differences $m_{j+1}-m_{j}$ there is a greatest one.

Applying this lemma to the case when $b_{j}=1 / \beta_{j}$ and $\nu=\gamma-1$, we have

$$
\left|k_{j}+m \beta_{j}\right| \leqq \delta \beta_{j} \quad(j=1,2, \cdots, \gamma-1) .
$$

Thence it follows that for every preassigned positive $\epsilon$ there exists a $\delta$ such that we now háve

$$
\left|h_{1}(t+2 m \pi)-h_{1}(t)\right| \leqq \sum_{k=0}^{\gamma-1}\left|c_{k} e^{i \beta_{k} t}\left(e^{2 \beta_{k} m \pi i}-1\right)\right|<\epsilon
$$

for all $t$ in the star-arm $A$. Let $R$ be a rectangle two sides of which are on the boundaries of $A$ and let it be subject to the condition that $h_{1}(t)$ does not vanish in $R$. Let $\epsilon$ be such that $\left|h_{1}(t)\right|>2 \epsilon$ in $R$. Then

$$
\left|h_{1}(t+2 m \pi)\right|>\epsilon
$$

when $t$ is in $R$ and $m$ is an integer admitted by the foregoing lemma.

We now return to the original form of $h(t)$ as given in (1.17). On each arm of the star associated with $h(t)$ we now take a rectangle $R$ obtained from the foregoing one by reversing the rotation by which the corresponding arm is put in the special position employed in the preceding argument; or, we take any rectangle $R$ on the arm and in which $h(t)$ does not vanish, in case the situation is 'such that the preceding argument reaches the goal before the introduction of Lemma 1.2 and the rectangle $R$. Then $h(t)$ is bounded away from 0 on $R$ and on all congruent rectangles (except a finite number at most) similar to those in the preceding paragraph and containing the points $t+2 m \pi$ with $t$ on $R$ and $m$ determined as in the lemma or $m$ sufficiently large when the lemma is not needed. 
A part of the foregoing results may be stated in the following lemma:*

LEMMA 1.3. There exists a positive number $\epsilon$ such that $|h(t)|>\epsilon$ for all large $t$ in sectors $S$ and for all large $t$ in rectangles $R$ or rectangles obtained from them by the translations $t^{\prime}=t+2 m \pi$ where $t$ is in $R$ and where $m$ is an integer admitted by Lemma 1.2 for the star-arm in question or $m$ is any sufficiently large integer in the cases where Lemma 1.2 is not employed in the argument.

For use in integrations later to be performed let us define a set of contours $\Gamma_{1}, \Gamma_{2}, \cdots$, passing through no zero of $h(t)$, such that 0 is interior to $\Gamma_{1}$ while $\Gamma_{i}$ is interior to $\Gamma_{j+1}$ and such that for $r$ greater than some preassigned number the distance from 0 to a point of $\Gamma_{r}$ is not less than $r$ and not greater than $r+\beta$ where $\beta$ is a sufficiently large given positive number, each contour having the property that it consists of circular arcs (with 0 as center) in the sectors $S$ and segments of the boundaries of the star-arms and straight line segments crossing these arms in the rectangles $R$ or such rectangles congruent to them as are admitted by Lemma 1.3 and the preceding discussion. Then the length of $\Gamma_{r}$ bears a bounded ratio to $2 \pi r$.

From Lemma 1.3 we then have the following:

Lemma 1.4. There exists a positive number $\epsilon$ such that $|h(t)|>\epsilon$ for every $t$ on every contour $\Gamma_{1}, \Gamma_{2}, \cdots$.

From the distribution of the numbers $m_{j}$ as described in Lemma 1.2, it follows that the contours $\Gamma_{1}, \Gamma_{2}, \cdots$ may be further restricted so that there exists a number $p$ such that no more than $p$ of the contours cross a given rectangle congruent to a given rectangle $R$ in accordance with Lemma 1.3.

1.8. Solution of equation (1.11). In equation (1.11) we take $a_{0}=0$, as we may do without loss of generality. We now propose to show that, when $\phi(x)$ is any given integral function, this equation has a solution $g(x)$ which is itself an integral function.

We denote by $G_{n}(x)$ the polynomial which satisfies the equation

$$
c_{0} G_{n}(x)+\sum_{k=1}^{\sigma} c_{k} G_{n}\left(x+a_{k}\right)=x^{n}
$$

and is (sometimes more precisely) defined by the formula

$$
G_{n}(x)=\frac{n !}{2 \pi i} \int_{C} \frac{e^{x t}}{h(t)} \frac{d t}{t^{n+1}},
$$

* For such results as those in Lemmas 1.3 and 1.4 see the address of R. E. Langer, Bulletin of the American Mathematical Society, vol. 37 (1931), pp. 213-239, and the papers there cited, especially those of J. D. Tamarkin. 
where $n$ is a positive integer or zero, $h(t)$ denotes the function defined in (1.17) and $C$ is a contour inclosing the point 0 and no singularity of the integrand other than $t=0$.

Let $\Gamma_{r}$, where $r$ is any positive integer, denote the contour represented by this symbol in the latter part of $\$ 1.7$. Form the function

$$
G_{n, r}(x)=\frac{n !}{2 \pi i} \int_{\Gamma_{r}} \frac{e^{x t}}{h(t)} \frac{d t}{t^{n+1}} .
$$

This function satisfies the equation

$$
c_{0} G_{n, r}(x)+\sum_{k=1}^{\sigma} G_{n, r}\left(x+a_{k}\right)=x^{n} .
$$

Let $x$ be now confined to any preassigned finite region $T$ of the $x$-plane. Then we have

$$
\left|G_{n, r}(x)\right| \leqq \frac{n !}{2 \pi} M_{1} \int_{\Gamma_{r}} \frac{\left|e^{x t}\right||d t|}{|t|^{n+1}}
$$

where $M_{1}$ is a dominant of $|1 / h(t)|$ for all $t$ on all contours $\Gamma_{r}$, the existence of this dominant being assured by Lemma 1.4. From the character of the contours $\Gamma_{r}$, as described in the latter part of $\$ 1.7$, we now see that a constant $M$ (independent of $x$ and $n$ and $r$ ) exists such that for all $x$ in $T$ we have

$$
\left|G_{n, r}(x)\right|<M \cdot n ! \cdot \rho^{r+\beta \cdot} \cdot r^{-n},
$$

where $\rho$ is such that $\rho>e^{|x|}$ for all $x$ in $T$.

Write the power series expansion of $\phi(x)$ in the form

$$
\phi(x)=\sum_{\nu=0}^{\infty} \lambda_{\nu} x^{\nu}
$$

Form the function $g(x)$,

$$
g(x)=\sum_{\nu=0}^{\infty} \lambda_{\nu} G_{\nu, \nu}(x) .
$$

Then the $(\nu+1)$ th term of the series here written is, in the region $T$ and for sufficiently large values of $\nu$, less in absolute value than the quantity

$$
M \cdot \nu ! \nu^{-v} \rho^{\nu+\beta}\left|\lambda_{\nu}\right| \text {. }
$$

As $\nu$ becomes infinite the superior limit of the $\nu$ th root of this quantity is zero since $\left|\lambda_{\nu}\right|^{1 / v}$ has the superior limit zero owing to the fact that $\phi(x)$ is an integral function. Therefore the series in (1.23) converges absolutely and uni- 
formly in any whatever preassigned finite region $T$. Since each term of this series is analytic throughout the finite plane it follows that $g(x)$ is itself an integral function.

It is readily verified by a direct substitution and a use of the named properties of $G_{v, \nu}(x)$ that this function $g(x)$ satisfies equation (1.11) with $a_{0}=0$.

We are thus led to the following theorem:

THEOREM 1.3. If $\phi(x)$ denotes the integral function defined in (1.22) then the series

$$
\sum_{\nu=0}^{\infty} \lambda_{\nu} G_{\nu, r}(x)
$$

is for suitable values of $r$ absolutely and uniformly convergent in every finite region of the complex plane (the value $r=\nu$ being always suitable) and defines $a$ sum function $g(x)$ which is an integral function of $x$ and satisfies equation (1.11) with $a_{0}=0$.

If $\phi(x)$ is further restricted to be of exponential type $q$ then it is easy to show (compare \$1.6) that $r$ may be given a sufficiently large fixed value (independent of $\nu$ ) in series (1.24) to insure convergence of the character indicated in the theorem. In fact, $\Gamma_{r}$ may be replaced by the circle $C_{r}$ of $\$ 1.6$. Then the resulting solution $g(x)$ of (1.11) is of exponential type not exceeding $r$ where $r$ is the radius of the circle $C_{r}$. By taking $r$ sufficiently small it may be brought about that the resulting solution $g(x)$ is of exponential type $q$; but there is no solution $g(x)$ of lower type than $q$. When $\phi(x)$ is of exponential type $q$ a solution $g(x)$ of (1.11) of exponential type $q$ may be called a principal solution of that equation.

1.9. The general case of (1.1) when the $\phi_{\nu}(x)$ are integral functions. In treating this case it is convenient to set forth first a particular solution of system (1.9). Again and without loss of generality we take $a_{0}=0$.

Form the functions

$$
g_{k}(x)=\frac{1}{2 \pi i} \sum_{j=0}^{\infty} \sum_{\nu=1}^{n} \int_{\Gamma} s_{\nu j}\left(e^{-x t} A_{\nu k} e^{x t}\right) \frac{e^{x t}}{h(t)} \frac{d t}{t^{j+1}} \quad(k=1,2, \cdots, n),
$$

where the coefficients $s_{v j}$ are those appearing in (1.13). Now the expression in parenthesis under the integral sign is a function of $t$. If one utilizes the form of this function of $t$ then by means of an easy modification of the argument employed in $\$ 1.8$ one may show that the series in (1.25) converge absolutely and uniformly in every preassigned finite region $T$ of the $x$-plane and that they define integral functions $g_{k}(x)$.

These integral functions may also be written in the form 


$$
g_{k}(x)=\frac{1}{2 \pi i} \sum_{j=0}^{\infty} \int_{\Gamma_{j}}\left(\sum_{v=1}^{n} s_{v j} A_{\nu k} e^{x t}\right) \frac{d t}{t^{j+1} h(t)} \quad(k=1,2, \cdots, n),
$$

the series having the same properties of convergence as before indicated.

Now by aid of (1.12) we have

$$
\begin{aligned}
\Delta g_{k}(x) & =\frac{1}{2 \pi i} \sum_{j=0}^{\infty} \int_{\Gamma_{j}} \sum_{\nu=1}^{n} s_{\nu j} A_{\nu k} e^{x t} \frac{d t}{t^{j+1}} \\
& =\sum_{\nu=1}^{n} A_{\nu k} \sum_{j=0}^{\infty} \frac{s_{\nu j}}{2 \pi i} \int_{\Gamma_{j}} e^{x t} \frac{d t}{t^{j+1}} \\
& =\sum_{\nu=1}^{n} A_{\nu k} \sum_{j=0}^{\infty} s_{\nu j} x^{j} / j !=\sum_{\nu=1}^{n} A_{\nu k} \phi_{\nu}(x)
\end{aligned}
$$

the last member being obtained from (1.13). Hence the functions $g_{k}(x)$ in (1.26) afford a solution of (1.9) with $a_{0}=0$.

That these same functions also afford a solution of (1.7) will next be proved. For this purpose substitute these functions $g_{k}(x)$ in the first member of (1.7) after replacing $\nu$ by $\mu$. Simplifying the result by aid of equation (1.8) and other preceding formulas we have

$$
\begin{aligned}
\sum_{k=1}^{n} c_{\mu k} E\left(a_{\mu k}\right) g_{k}(x) & =\frac{1}{2 \pi i} \sum_{j=0}^{\infty} \int_{\Gamma_{j}} \sum_{\nu=1}^{n} s_{\nu j}\left[\sum_{k=1}^{n} c_{\mu k} E\left(a_{\mu k}\right) A_{\nu k}\right] e^{x t} \frac{d t}{t^{j+1} h(t)} \\
& =\frac{1}{2 \pi i} \sum_{j=0}^{\infty} s_{\mu j} \int_{\Gamma_{j}} \frac{\Delta e^{x t} d t}{t^{j+1} h(t)} \\
& =\frac{1}{2 \pi i} \sum_{j=0}^{\infty} s_{\mu j} \int_{\Gamma_{j}} \frac{e^{x t} d t}{t^{j+1}}=\phi_{\mu}(x) .
\end{aligned}
$$

Hence system (1.7) is satisfied by these functions $g_{k}(x)$.

Thus we have the following theorem:

THEOREM 1.4. When the functions $\phi_{\nu}(x)$ in the non-singular system (1.7) are given integral functions and when the constant $a_{0}$ in (1.10) has the value 0 the system has a solution $g_{k}(x), k=1,2, \cdots, n$, consisting of integral functions defined by equations (1.26), and the series in these equations converge absolutely and uniformly in every preassigned finite region $T$ of the $x$-plane.

From this theorem it follows that every non-singular system (1.1) has a solution consisting of integral functions whenever the given functions $\phi_{\nu}(x)$ are themselves integral. The more special case in which the $\phi_{\nu}(x)$ are of exponential type has already been treated in $\$ 1.6$. 


\section{Simultaneous EXPANSIONS OF INTEGRAL FUNCTIONS IN COMPOSITE POWER SERIES}

2.1. Formulation of the problem. For $n>1$ we consider the question of expanding $n$ integral functions $f_{1}(x), f_{2}(x), \cdots, f_{n}(x)$ simultaneously in composite power series, that is, we consider the problem of representing these functions in the form

$$
f_{\nu}(x)=\sum_{k=0}^{\infty} \sum_{j=1}^{n} c_{j k}\left(x-a_{\nu j}\right)^{k} \quad(\nu=1,2, \cdots, n),
$$

where the coefficients $c_{j k}$ are to be independent of both $x$ and $\nu$. We impose the further condition on the coefficients $c_{j k}$ that they shall be such that the series in the equations

$$
g_{j}(x)=\sum_{k=0}^{\infty} c_{j k} x^{k} \quad(j=1,2, \cdots, n)
$$

shall converge for all finite values of $x$; then the sum functions $g_{j}(x)$ defined by them will be integral functions. These conditions on the $c_{j k}$ are equivalent to the conditions that the quantities $\left|c_{j k}\right|^{1 / k}, j=1,2, \cdots, n$, shall all have the limit zero as $k$ becomes infinite. Furthermore we subject the given constants $a_{v j}$ to the condition that the determinant $\Delta(t)$ whose element in $\nu$ th row and $j$ th column is $\exp \left(-a_{v j} t\right)$ shall not be identically zero as a function of $t$. In the exceptional or singular case in which this condition on $\Delta(t)$ is not satisfied the general investigation will require methods different from those here employed; and the results will lack the simplicity and elegance which. belong to the general case here treated.

For $n=1$ the problem evidently reduces to the classical problem of expansions in power series. We suppose throughout that $n>1$.

Under the conditions named we shall show that such simultaneous expansions always exist and indeed that they always exist subject to the further condition that the functions $g_{j}(x), j=1,2, \cdots, n$, shall be of exponential type provided in the latter case that the functions $f_{\nu}(x), \nu=1,2, \cdots, n$, are of exponential type.

If we employ the notation defined in (2.2) we may write (2.1) in the form

$$
f_{\nu}(x)=\sum_{j=1}^{n} g_{j}\left(x-a_{\nu j}\right) \quad(\nu=1,2, \cdots, n) .
$$

Integral solutions of this system evidently lead through (2.2) to the required expansions (2.1). The condition put on $\Delta(t)$ is just that which is required to make the results of the first part of this paper applicable to system (2.3) and hence to the expansion problem here set. 
2.2. Expansions in the case of general integral functions $f_{\nu}(x)$. From Theorem 1.4 and the remark following it one concludes that system (2.3) has in this case integral solutions $g_{j}(x), j=1,2, \cdots, n$. Therefore we have the following theorem:

THEOREM 2.1. If $f_{1}(x), f_{2}(x), \cdots, f_{n}(x)$ are any given integral functions and if the constants $a_{r j}$ are such that the determinant $\Delta(t)$ has the property described in the first paragraph of $\$ 2.1$, then these functions $f_{v}(x)$ have simultaneous expansions of the form (2.1) where

$$
\lim _{k=\infty}\left|c_{j k}\right|^{1 / k}=0 \quad(j=1,2, \cdots, n) .
$$

Formulas in $\$ 1.9$ afford an effective means of obtaining suitable coefficients $c_{j k}$ to be employed in the expansions (2.1). Only in exceptional cases is it true that these expansions are unique. The determination of the extent of arbitrary elements involved in the coefficients of the expansions depends on the (as yet undeveloped) theory of system (2.3) for the case when $f_{\nu}(x) \equiv 0$, $\nu=1,2, \cdots, n$.

2.3. Expansions when the $f_{v}(x)$ are of exponential type. Applying Theorem 1.2 to system (2.3) in the case when the functions $f_{v}(x)$ are of exponential type and interpreting the results in terms of the expansions in (2.1), we have the following theorem:

TheOREM 2.2. If the functions $f_{1}(x), f_{2}(x), \cdots, f_{n}(x)$ are of exponential type not exceeding $q$, one at least of them being precisely of type $q$, and if the constants $a_{v j}$ are such that the determinant $\Delta(t)$ has the property described in the first paragraph of $\$ 2.1$, then the functions $f_{v}(x)$ have simultaneous expansions of the form (2.1) such that the associated functions $g_{j}(x)$ of (2.2) are of exponential type and indeed such that these functions $g_{j}(x)$ are of exponential type not exceeding $q$, one at least of them being precisely of type $q$.

When the associated $g_{j}(x)$ are of exponential type not exceeding $q$ we shall say that the series in (2.1) afford principal expansions of the functions $f_{\nu}(x)$.

Even with the strongest conditions imposed on the coefficients $c_{j k}$ by the latter part of the foregoing theorem it is still true that the expansions (2.1) need not be unique. In all cases belonging to this section possible values of the coefficients $c_{j k}$ are readily determined from the special case of equation (1.16) applicable here, as we show in the next paragraph; and these values may well vary in dependence upon the radius $r$ of the circle $C_{r}$ appearing in (1.16). 
In connection with the expansions

$$
f_{\nu}(x)=\sum_{k=0}^{\infty} \alpha_{\nu k} x^{k} / k ! \quad(\nu=1,2, \cdots, n),
$$

form the functions

$$
F_{\nu}(t)=\sum_{k=0}^{\infty} \frac{\alpha_{\nu k}}{t^{k+1}} \quad(\nu=1,2, \cdots, n) .
$$

Let $\Delta_{\nu j}(t)$ be the cofactor of the element in the $\nu$ th row and $j$ th column of $\Delta(t)$. Then by aid of (1.16) it may readily be shown that suitable coefficients $c_{j k}$ in (2.1) are the following:

$$
c_{j k}=\frac{1}{2 \pi i(k !)} \int_{C_{r}}\left(\sum_{r=1}^{n} \Delta_{v j}(t) F_{\nu}(t)\right) \frac{t^{k} d t}{\Delta(t)},
$$

where $j=1,2, \cdots, n$ and $k=0,1,2, \cdots$.

2.4. The case $a_{\nu j}=a_{\nu \nu}$ for $\nu>j$. In this case system (2.3) is equivalent to the system consisting of the first equation in (2.3) and the following $n-1$ equations:

$$
f_{v-1}(x)-f_{v}(x)=\sum_{j=\nu}^{n}\left\{g_{j}\left(x-a_{v-1, j}\right)-g_{j}\left(x-a_{v j}\right)\right\} \quad(\nu=2,3, \cdots, n)
$$

In case $a_{n-1, n}=a_{n n}$ it is clear that we must have $f_{n-1}(x)=f_{n}(x)$ as a necessary condition for satisfying the system. In fact, it is easy to see that the functions $f_{\nu}(x)$ must satisfy one or more special restrictive conditions if one or more of the relations

$$
a_{\nu-1, \nu}-a_{\nu p} \neq 0 \quad(\nu=2,3, \cdots, n)
$$

fails to be satisfied. But if conditions (2.5) are all satisfied then we have an instance of the general theory already developed; we shall suppose that these conditions are satisfied. We assume that the given functions $f_{1}(x), \cdots, f_{n}(x)$ are all integral functions. We require that the functions $g_{1}(x), \cdots, g_{n}(x)$ shall be integral functions.

Taking $\nu=n$ in (2.4) we see that $g_{n}(x)$ is uniquely determined as an integral function except for an arbitrary additive periodic integral function of period $a_{n-1, n}-a_{n n}$. Taking $g_{n}(x)$ to be any integral function satisfying (2.4) for $\nu=n$ we may then determine $g_{n-1}(x)$ uniquely except for an additive integral function of period $a_{n-2, n-1}-a_{n-1, n-1}$. With $g_{n-1}(x)$ determined we proceed similarly to the determination of $g_{n-2}(x)$, and we continue thus until $g_{2}(x)$ is determined. Then the first equation in (2.3) uniquely determines $g_{1}(x)$. It appears, therefore, that in the present case one can determine com- 
pletely the arbitrary elements in the solution of (2.3) subject to the named conditions. Hence all possible expansions (2.1) are completely determined for the present case.

If we further restrict the given functions $f_{1}(x), \cdots, f_{n}(x)$ to be of exponential type not greater than $q$ we may likewise determine the functions $g_{1}(x), \cdots, g_{n}(x)$ so that they are of exponential type not greater than $q$ and we may show precisely what is arbitrary in the determination of such functions subject to these conditions. These results may then be carried over to the corresponding case of the expansions (2.1).

There is one case of particular interest in which the expansions (2.1), when subject to the condition named in the preceding paragraph, are unique except for the trivial restriction that the constants $c_{j 0}, j=1,2, \cdots, n$, are not separately determined but only their sum is determined. This is the case in which the functions $f_{1}(x), \cdots, f_{n}(x)$ are of exponential type not greater than $q$ while at the same time the relations

$$
q\left|a_{\nu-1, \nu}-a_{\nu \nu}\right|<2 \pi \quad(\nu=2,3, \cdots, n)
$$

are all satisfied. For in this case each $g_{j}(x)$ is uniquely determined except for an additive constant. These conditions are obviously satisfied whenever inequalities (2.5) hold provided that $q=0$ and in particular provided that the functions $f_{v}(x)$ are polynomials.

2.5. The case $n=2$. For the case $n=2$ system (2.3) may be written in the form

$$
\begin{aligned}
& f_{1}\left(x+a_{11}\right)=g_{1}(x)+g_{2}\left(x+a_{11}-a_{12}\right), \\
& f_{2}\left(x+a_{21}\right)=g_{1}(x)+g_{2}\left(x+a_{21}-a_{22}\right) .
\end{aligned}
$$

The exceptional case here is that in which $a_{11}-a_{12}=a_{21}-a_{22}$. When this condition is satisfied, the system can have a solution only when $f_{1}\left(x+a_{11}\right)$ $=f_{2}\left(x+a_{21}\right)$, as one sees from (2.7); and in this case it is clear that either of the integral functions $g_{1}(x)$ and $g_{2}(x)$ may be assigned at will and that the other is then uniquely determined: the case is therefore trivial.

When $a_{11}-a_{12} \neq a_{21}-a_{22}$ the case belongs to that treated in $\$ 2.4$.

As an application of the case when $a_{11}=a, a_{12}=b, a_{21}=0=a_{22}$, where $a \neq b$, we see that an arbitrary integral function $f(x)$ may be expanded in the form

$$
f(x)=\sum_{k=0}^{\infty}\left\{c_{k}(x-a)^{k}+\gamma_{k}(x-b)^{k}\right\}
$$

where the sums $c_{k}+\gamma_{k}, k=0,1,2, \cdots$, have any preassigned values subject to the condition that

$$
\lim _{k=\infty}\left|c_{k}+\gamma_{k}\right| 1 / k
$$


shall exist and be equal to zero; and the parts of $f(x)$ represented by the component power series in $x-a$ and $x-b$ respectively, when these parts are themselves required to be integral functions, are unique except for an arbitrary integral periodic function of period $a-b$ to be added to one part and subtracted from the other.

Furthermore, if $f(x)$ is of exponential type not greater than $q$ and if the parts of $f(x)$ represented by the component power series in $x-a$ and $x-b$ respectively are required to be of exponential type not greater than $q$, then there exists an expansion of the form (2.8) subject to the condition that

$$
\underset{k=\infty}{\lim \sup }\left|\left(c_{k}+\gamma_{k}\right) / k !\right|^{1 / k} \leqq q ;
$$

and the expansion is unique except for an arbitrary periodic function of period $a-b$ and of exponential type not greater than $q$, such periodic function to be added to one component part of $f(x)$ and subtracted from the other. If we add the further restriction that $q|a-b|<2 \pi$ then this periodic function reduces to a constant, so that the expansion (2.8) is then essentially unique.

2.6. Generalizations. From the fact established in $\$ 1.9$ that the nonsingular system (1.1) always has integral solutions when the $\phi_{\nu}(x)$ are given integral functions it follows that any set $\phi_{1}(x), \cdots, \phi_{n}(x)$ of integral functions has simultaneous expansions in the form

$$
\phi_{\nu}(x)=\sum_{k=0}^{\infty} \sum_{j=1}^{n} \alpha_{k j} c_{\nu j}\left(x+a_{\nu j}\right)^{k} \quad(\nu=1,2, \cdots, n)
$$

where the constants $\alpha_{k j}$ are independent of $x$ and $\nu$ and where the component functions $g_{j}(x)$,

$$
g_{j}(x)=\sum_{k=0}^{\infty} \alpha_{k j} x^{k} \quad(j=1,2, \cdots, n),
$$

are themselves integral functions. If the $\phi_{\nu}(x)$ are subject to the further condition that they shall be of exponential type not greater than $q$ then the expansions (2.9) exist subject (as one sees from \$1.6) to the condition that the component functions $g_{j}(x)$ shall also be of exponential type not greater than $q$. If furthermore at least one of the functions $\phi_{\nu}(x)$ is of precisely type $q$ then one at least of the component functions $g_{j}(x)$ is of precisely type $q$.

These results are capable of extension by means of the generalizations indicated near the end of $\$ 1.1$.

There is a special case arising from expansions (2.9) to which particular attention may be directed. Let $a_{1 j}=-a_{j}, j=1,2, \cdots, n$, where $a_{1}, a_{2}, \cdots$, 
$a_{n}$ are different constants, and let the other $a_{v j}$ have the value 0 . Let $c_{1 j}=1$, $j=1,2, \cdots, n$, while the other $c_{v i}$ are such that the matrix

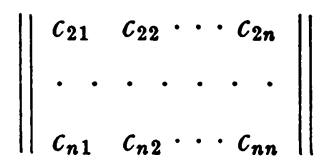

is of rank $n-1$. Then the corresponding system (1.1) is non-singular. Consider the problem of expanding a given integral function $\phi(x)$ in the form

$$
\phi(x)=\sum_{k=0}^{\infty} \sum_{j=1}^{n} \alpha_{k j}\left(x-a_{j}\right)^{k} .
$$

Since $\phi(x)$ thus takes the place of $\phi_{1}(x)$ in (2.9) and since the remaining integral functions

$$
\phi_{2}(x), \cdots, \phi_{n}(x)
$$

in (2.9) may be assigned at will, it follows that an expansion of the form (2.10) exists (not necessarily unique) such that

while the quantities

$$
\lim _{k=\infty}\left|\alpha_{k j}\right|^{1 / k}=0 \quad(j=1,2, \cdots, n),
$$

$$
\beta_{\nu k}=\sum_{j=1}^{n} e_{\nu j} \alpha_{k j} \quad(\nu=2, \cdots, n ; k=0,1,2, \cdots)
$$

may be assigned at will subject to the condition that

$$
\lim _{k=\infty}\left|\beta_{\nu k}\right|^{1 / k}=0 \quad(\nu=2, \cdots, n) .
$$

This result affords an interesting generalization of the Cauchy-Taylor expansion of an integral function. Whether there exists a corresponding generalization for functions analytic in a finite region $I$ have not sought to determine.

If $\phi(x)$ is further restricted to be of exponential type not greater than $q$ then there exists an expansion of the form (2.10) (not necessarily unique) such that

$$
\limsup _{k=\infty}\left|\alpha_{k_{i}} / k !\right|^{1 / k} \leqq q \quad(j=1,2, \cdots, n),
$$

while the quantities

$$
\beta_{\nu k} \quad(\nu=2, \cdots, n ; k=0,1,2, \cdots)
$$

may be assigned at will subject to the condition that

$$
\limsup _{k=\infty}\left|\beta_{\nu k} / k !\right|^{1 / k} \leqq q \quad(\nu=2, \cdots, n) .
$$




\section{EXPANSIONS IN SERIES OF EXPONENTIAL FUNCTIONS}

3.1. Properties of exponential sums. Let us denote by $h(t)$ the function

$$
h(t)=c_{1} e^{a_{1} t}+c_{2} e^{a_{2} t}+\cdots+c_{n} e^{a_{n} t}, \quad n>1,
$$

where $a_{1}, a_{2}, \cdots, a_{n}$ are different constants and $c_{1}, c_{2}, \cdots, c_{n}$ are constants different from zero. And let us consider the problem of bounding away from zero the function $e^{-x t} h(t)$ for suitable given values of $x$ and for suitable ranges of $t$. The results are needed for our later investigation (\$3.2) of certain contour integrals.

Let $P$ be the smallest convex polygon, in the complex plane, containing the points $a_{1}, a_{2}, \cdots, a_{n}$; this polygon may in special cases reduce to a straight line segment. Let $Q$ be the polygon* obtained by reflecting $P$ through the real axis. For the sake of definiteness we suppose that the notation is so chosen that the vertices of $P$, taken in counter-clockwise order, are $a_{1}, a_{2}$, $\cdots, a_{\nu}(\nu \leqq n)$ and that no $a_{j}$ has its real part less than that of $a_{1}$. Moreover we suppose that the vertices are so taken that no three of these $a$ 's at the vertices lie on the same straight line. Let $l_{1}, l_{2}, \cdots, l_{\nu}$ be the rays normal to the sides of $Q$ at their centers and drawn outward from this polygon; when $Q$ reduces to a straight line it is to be understood that these rays are two in number and that they are drawn so that there is one in each direction from the middle point of the line. We take the notation so that $l_{1}, l_{2}, \cdots, l_{v}$ are in clockwise order and so that $l_{1}$ is the normal to the side joining the conjugates of $a_{1}$ and $a_{2}$.

Let $a_{j}$ and $a_{k}$ be two consecutive vertices of $P$ and let $l_{\mu}$ be the normal to that side of $Q$ which joins the corresponding vertices of $Q$. If $R(z)$ denotes the real part of $z$, then the line $R\left(a_{j} t\right)=R\left(a_{k} t\right)$ is parallel to the line $l_{\mu}$. Let $\rho$ be a positive number whose value is later to be conveniently restricted. On each side of each line $l_{1}, l_{2}, \ldots, l_{\nu}$ and at a distance $\rho$ from it draw a ray in such a way that these rays will make a sort of infinite star similar to that considered in $\S 1.7$ and containing the rays $l_{1}, l_{2}, \ldots, l_{\nu}$ in the centers of its arms. These rays form certain sectors $S$, similar to those in $\$ 1.7$ and containing no interior points of the named infinite star.

In order to have sectors exactly like those in $\$ 1.7$ it is necessary to divide some of the sectors $S$ into smaller sectors by excluding other strips; but this further division is to serve only a temporary purpose in the argument. It may be described as follows. Let $m_{1}, m_{2}, \cdots, m_{\nu}$ be rays from zero to infinity parallel to $l_{1}, l_{2}, \cdots, l_{v}$ respectively but such that $m_{k}$ goes to infinity in a direction opposite to that of $l_{k}$. Some rays $m_{k}$ may go to infinity in the same

* Such polygons as $P$ and $Q$ have been employed by Pólya, Mathematische Annalen, vol. 89 (1923), pp. 179-191. 
direction as other rays $l_{j}$ (and they will do so when $Q$ has pairs of parallel sides); remove such rays $m_{k}$; if any rays $m_{\sigma}$ remain after this removal, denote them by $m_{\alpha}, m_{\beta}, \cdots$. Along the rays $m_{\alpha}, m_{\beta}, \cdots$ remove strips of width $2 \rho$ as in the case of the preceding paragraph. Then some sectors $S$ are separated into two or more sectors (together with one or more strips). After all such separations are made; let $S^{\prime}$ be a symbol to denote the totality of sectors obtained, including undivided sectors $S$ and the parts into which some sectors $S$ have been separated.

From Lemma 1.1 it follows that $\rho$ may be taken sufficiently large that $h(t)$, and hence $e^{-x t} h(t)$, shall have no zero in any sector $S^{\prime}$. Moreover, from the same lemma it follows that $\rho$ may be taken sufficiently large (and we so take it) that $e^{-x t} h(t)$ is bounded away from zero in the sectors $S^{\prime}$ when $x$ is any one of the points $a_{1}, a_{2}, \cdots, a_{n}$. In fact, when $x$ has any such value the function $e^{-x t} h(t)$ is a function meeting the conditions on $h(t)$ in $\$ 1.7$ so that Lemmas 1.3 and 1.4 are also applicable to $e^{-x t} h(t)$ for such values of $x$.

Let $a_{j}, a_{k}$ and $a_{l}$ be any three consecutive vertices of $P$ in counterclockwise order and let $l_{j}$ and $l_{k}$ be the rays perpendicular to the sides of $Q$ with corresponding vertices. Let $S_{j k}$ be the sector $S$ lying between $l_{j}$ and $l_{k}$. Suppose that $t$ varies in $S_{j k}$. Let $x$ be a fixed point in $P$. We have

$$
\left|e^{-x t} h(t)\right|=\left|e^{\left(a_{k}-x\right)\left(t-\bar{a}_{k}\right)}\right| \cdot\left|e^{\bar{a}_{k}\left(a_{k}-x\right)}\right| \cdot\left|e^{-a_{k} t} h(t)\right|,
$$

where $\bar{a}_{k}$ is the conjugate of $a_{k}$. The last factor in the second member is bounded away from zero for large $t$ in the named sector, as we have already seen. The middle factor is a constant different from zero, since $x$ is fixed. The argument of the exponent of the first factor lies between $-\frac{1}{2} \pi$ and $\frac{1}{2} \pi$ inclusive, as one may readily show graphically, if (as we do by taking $\rho$ sufficiently large) we restrict the sector $S_{j k}$ to lie in the sector formed by rays from $\bar{a}_{k}$ to infinity in the direction of the rays $l_{j}$ and $l_{k}$ : in establishing the named fact it is convenient temporarily to transform the points of the plane by adding $-\bar{a}_{k}$ to each value in it so that the representation of $\bar{a}_{k}$ becomes the point zero and then to begin from the plots of $\bar{x}-\bar{a}_{k}$ and $t-\bar{a}_{k}$. Thence it follows that $e^{-x t} h(t)$ is bounded away from zero in the named sector. Furthermore it follows from Lemma 1.3 that $e^{-x t} h(t)$ is bounded away from zero for all large $t$ in rectangles congruent to the rectangles $R$ in the way specified in that lemma, these rectangles $R$ being chosen with reference to the function $e^{-x t} h(t)$.

Let us now further restrict $x$ to lie in the interior of $P$. Then there exists a positive number $\epsilon$ such that

$$
-\frac{1}{2} \pi+\epsilon \leqq \arg \left\{\left(a_{k}-x\right)\left(t-\bar{a}_{k}\right)\right\} \leqq \frac{1}{2} \pi-\epsilon .
$$


Thence it follows that the function $t^{-1} e^{-x t} h(t)$ is bounded away from zero as $t$ becomes infinite in the named sector.

The same function is also bounded away from zero if $x$ is on the boundary of $P$ but not at $a_{k}$ while $t$ becomes infinite in the named sector in such a way as to remain outside of each of two parabolas with vertex at $\bar{a}_{k}$ and having the named rays from $\bar{a}_{k}$ parallel to $l_{j}$ and $l_{k}$ as their principal diameters.

It may now be observed that every strip along one of the rays $m_{\alpha}, m_{\beta}, \cdots$ lies (except for a finite part of it) entirely in a sector $S$ and that it has a direction intermediate to the directions of the bounding rays of this sector $S$. Thence it follows also that such a strip (except for a finite part of it) lies entirely outside of the parabolas along the bounding rays of this sector $S$. Hence the strips along the rays $m_{\alpha}, m_{\beta}, \cdots$ may be removed and we thus return to the set of sectors $S$ as defined in this section; and for the plane so divided we have the requisite character of $e^{-x t} h(t)$ or $t^{-1} e^{-x t} h(t)$ as a function bounded away from zero, in accordance with the paragraph next following.

Summing up these results we may state that $e^{-x t} h(t)$ is bounded away from zero for any given $x$ in $P$ and for all large $t$ in all sectors $S$ formed with sufficiently large $\rho$ and in all rectangles congruent to rectangles $R$ in accordance with Lemma 1.3; that $t^{-1} e^{-x t} h(t)$ is bounded away from zero for each interior point $x$ of $P$ and for all large $t$ in all such sectors $S$; and that $t^{-1} e^{-x t} h(t)$ is bounded away from zero for each $x$ on the boundary of $P$ and not at a vertex of $P$ and for all large $t$ in all such sectors $S$ and outside of all parabolas of the sort described for $\bar{a}_{k}$ in the previous paragraph, two such parabolas being formed at each vertex of $Q$.

3.2. Properties of certain contour integrals. Let $C_{1}, C_{2}, \ldots, C_{s}, \ldots$ be a set of different contours in the complex plane such that any given point on $C_{j}$ is either interior to $C_{j+1}$ or on $C_{j+1}$ and such that for every $s$ there exists an $r$ such that the contour $C_{s}$ is a contour $\Gamma_{r}$ of the sort described in $\$ 1.7$ and suitable to apply to $e^{-x t} h(t)$ for points $x$ in $P$ as the contours $\Gamma_{r}$ apply to the function $h(t)$ of $\$ 1.7$ and such that for every $r$ there is an $s$ such that $C_{s}$ is a contour $\Gamma_{r}$.

Let $\psi(t)$ be any function of $t$ which is analytic at infinity and vanishes there and let us write

$$
\psi(t)=\gamma_{1} / t+\gamma_{2} / t^{2}+\gamma_{3} / t^{3}+\cdots,|t|>q .
$$

Let $r$ be a fixed integer such that the contour $C_{r}$ lies entirely within the region of convergence of the series in (3.2). Form the function $F_{r}(x)$,

$$
F_{r}(x)=\frac{1}{2 \pi i} \int_{C_{r}} e^{x t}\{h(t)\}^{-1} \psi(t) d t .
$$

Then $F_{r}(x)$ is a function of exponential type; and, in fact, it is such a function 
as arises from the solution of equation (1.11) when $\phi(x)$ is a given function of exponential type, as one sees from Part I and especially from $\$ 1.6$.

Let $p$ be any positive integer and form the function $F_{r+p}(x)$ by changing $r$ to $r+p$ in (3.3). We shall show that

$$
\lim _{p=\infty} F_{r+p}(x)=0
$$

when any one of the following conditions is satisfied:

(1) when $x$ is in the interior of $P$;

(2) when $x$ is on the boundary of $P$ and is not a vertex of $P$;

(3) when $x$ is a vertex of $P$ provided in this case that $\gamma_{1}=0$.

It is convenient to carry out the proof first for the case when $\gamma_{1}=0$. Then a number $M$ exists such that $\left|t^{-2} \psi(t)\right|<M$ on all the contours $C_{r+p}$. We let $x$ be any point of $P$ either in the interior or anywhere on the boundary. Then from the results at the end of $\$ 3.1$ it follows that a constant $M_{1}$ exists such that $\left|e^{x t}\{h(t)\}^{-1}\right|<M_{1}$. Hence there is a constant $M_{2}$ such that in this case we have

$$
\left|F_{r+p}(x)\right|<M_{2} \int_{C_{r+p}}|t|^{-2}|d t| .
$$

This implies the truth of (3.4) when $\gamma_{1}=0$ and $x$ is anywhere in $P$.

With this result in hand we see that (3.4) will be established in the three cases (1), (2), (3) if we further prove its validity in cases (1) and (2) for the particular function $\psi(t)=1 / t$, since we may then pass to the general case in an obvious manner.

In case (1) let us write

$$
F_{r+p}(x)=\frac{1}{2 \pi i}\left(\int_{S_{r+p}}+\int_{A_{r+p}}\right) e^{x t}\{h(t)\}^{-1} t^{-1} d t,
$$

where $S_{r+p}$ denotes the set of paths consisting of the parts of $C_{r+p}$ which lie in the sectors $S$ while $A_{r+p}$ is the set of paths consisting of the remaining parts of $C_{r+p}$. Then on $A_{r+p}$ the integrand has a dominant of the form $M /|t|$ while on $S_{r+p}$ it has a dominant of the form $M /\left|t^{2}\right|$, as one sees from the results in the last paragraph of $\$ 3.1$. Thence we conclude readily to the truth of (3.4) for the present case, since the total length of the parts $A_{r+p}$ is bounded.

In case (2) we may use notationally the same equation (3.5) where we now understand that $A_{r+p}$ denotes the set of paths consisting of the parts of $C_{r+p}$ which lie in the parabolas described near the end of $\$ 3.1$ while $S_{r+p}$ consists of the remaining parts of $C_{r+p}$. The conclusion that (3.4) is valid in the present case is reached in the same way as in the preceding paragraph but by using 
the additional fact that the total length of the parts $A_{r+p}$ bears to the minimum distance $d$ from zero to points of $A_{r+p}$ a ratio which is infinitesimal as $r+p$ becomes infinite.

Thus the relation (3.4) is established for all points $x$ of $P$ except that when $x$ is at a vertex of $P$ we require that $\gamma_{1}$ shall have the value zero.

3.3. Expansions in series of exponential functions. Let $S_{r+p}(x)$ denote the negative of the sum of the residues of the function $e^{x t}\{h(t)\}^{-1} \psi(t)$ in the region bounded by the contours $C_{r+p-1}$ and $C_{r+p}$. If the function has no singularity in this region we shall understand that $S_{r+p}(x)$ is identically zero. In all other cases $S_{r+p}(x)$ is a function of the form $c e^{\alpha x}$ or a sum of a finite number of such functions. We have

$$
F_{r}(x)-F_{r+p}(x)=\sum_{k=1}^{p} S_{r+k}(x) .
$$

If we suppose that $x$ is a point of $P$ and in case $\gamma_{1} \neq 0$ that it is not a vertex of $P$ then relation (3.4) is applicable to the foregoing equation when $p$ is allowed to become infinite and we have the following theorem:

THEOREM 3.1. The function $F_{r}(x)$ defined in (3.3) has the expansion

$$
F_{r}(x)=\sum_{k=1}^{\infty} S_{r+k}(x)
$$

in series of exponential functions, valid for all values of $x$ in the polygon $P$, except that the vertices are to be excluded when $\gamma_{1} \neq 0$.

In the special case when $h(t)=e^{t}-1$ the series in (3.6) is a Fourier series. The polygon $P$ in this case reduces to the interval (01) of the real axis, the end points of the interval serving as the vertices of the polygon. A further treatment of Fourier series from this point of view will appear in a forthcoming paper in Annals of Mathematics.

The foregoing theorem serves to expand in series (3.6) any whatever function that may be put in the form (3.3). If $h(0) \neq 0$ it is evident that any given polynomial in $x$ may be put in the form $F_{1}(x)$ by taking $C_{1}$ to be a small circle about 0 as a center and by choosing $\psi(t)$ properly as a polynomial in $1 / t$. The function $F_{1}(x)+$ constant may also in other cases represent any whatever polynomial in $x$. Hence, in particular, all polynomials have expansions in the form (3.6), or in this form with an additive constant, valid in polygons $P$ as indicated.

3.4. A special class of the foregoing expansions. We shall now examine the special case of the foregoing expansion theory in which the function $e^{x t}\{h(t)\}^{-1}$ has the form 


$$
\frac{e^{x t}}{\left(e^{\rho_{1} t}-1\right)\left(e^{\rho_{2} t}-1\right) \cdots\left(e^{\rho_{n} t}-1\right)}
$$

where $\rho_{1}, \rho_{2}, \cdots, \rho_{n}$ are $n$ real or complex constants different from 0 and such that neither the sum nor the difference of two of them is zero.

It is convenient, for the sake of simplicity, to normalize the problem by means of certain elementary transformations. If $\rho_{k}$ has a negative real part we may replace $\rho_{k}$ by $-\rho_{k}$ by multiplying both numerator and denominator in (3.7) by $-e^{-\rho_{k} t}$ and so obtain (except for an irrelevant change in sign) a similar expression with $x$ replaced by $x-\rho_{k}$; by a translation in the $x$-plane we may then replace $x-\rho_{k}$ by $x$. We suppose all such translations made so that we shall assume that the real part of each $\rho_{k}$ is positive or zero. Then the further conditions on $\rho_{1}, \rho_{2}, \cdots, \rho_{n}$ are that they are different from each other and from zero. Then the point zero is on the boundary of the polygon $P$, introduced (\$3.1) in the general case, and the greatest real value of a point in $P$ is the sum of the real parts of $\rho_{1}, \rho_{2}, \cdots, \rho_{n}$. We suppose that the notation is so chosen that

$$
-\frac{1}{2} \pi \leqq \arg \rho_{1} \leqq \arg \rho_{2} \leqq \cdots \leqq \arg \rho_{n} \leqq \frac{1}{2} \pi .
$$

By means of a straight line join each point (except the last) in the set

$$
\begin{gathered}
0, \rho_{1}, \rho_{1}+\rho_{2}, \rho_{1}+\rho_{2}+\rho_{3}, \cdots, \rho_{1}+\rho_{2}+\cdots+\rho_{n}, \\
\rho_{2}+\cdots+\rho_{n}, \cdots, \rho_{n-1}+\rho_{n}, \rho_{n}, 0
\end{gathered}
$$

to the one which follows it, thus forming a convex polygon of an even number of sides and having its sides parallel in pairs. This is the polygon $P$, as one sees by examining points $x$ in the sectors formed by adjacent sides. Then the points $x$ in $P$ are the points

$$
x=\lambda_{1} \rho_{1}+\lambda_{2} \rho_{2}+\cdots+\lambda_{n} \rho_{n} \quad\left(0 \leqq \lambda_{k} \leqq 1 ; k=1,2, \cdots, n\right),
$$

as one sees by aid of the fact that each of these points lies in the strips each of which is bounded by two parallel sides of $P$ and by showing that every point in $P$ is a point $x$ of the named form. The boundary of $P$ is traced out in counter-clockwise order by starting with all $\lambda$ 's equal to zero, then letting $\lambda_{1}$ increase from 0 to 1 , then $\lambda_{2}$ from 0 to 1 , and so on to $\lambda_{n}$ letting it increase from 0 to 1 , then letting $\lambda_{1}$ decrease from 1 to $0, \lambda_{2}$ from 1 to 0 , and so on till $\lambda_{n}$ decreases from 1 to 0 .

For every point $x$ in $P$ the function (3.7) may be written in the form

$$
\frac{e^{x t}}{\left(e^{\rho_{1} t}-1\right) \cdots\left(e^{\rho_{n} t}-1\right)}=\frac{e^{\lambda_{1} \rho_{1} t}}{e^{\rho_{1} t}-1} \cdot \frac{e^{\lambda_{2} \rho_{2} t}}{e^{\rho_{2} t}-1} \cdot \cdots \frac{e^{\lambda_{n} \rho_{n} t}}{e^{\rho_{n} t}-1},
$$


where $0 \leqq \lambda_{k} \leqq 1, k=1,2, \cdots, n$. For this special case the inequalities obtained in $\$ 3.1$ may be derived in a very simple manner, as one may see by applying the methods of $\$ \S 1.7$ and 3.1 separately to each factor of the second member of (3.11) and simplifying the procedure in obvious ways for these special cases.

Moreover, when no two of the $\rho_{j}$ have a real ratio, the contours $C_{1}, C_{2}, \cdots$ may be chosen so that $C_{k}$ incloses just $k$ zeros of $h(t)$ for $k=1,2, \cdots$. Hence the terms $S_{r+k}(x)$ in (3.6) may all be taken in the form $c e^{\alpha x}$ so that we have to do with expansions of the form*

$$
F(x)=\alpha_{00}+\sum_{m=1}^{\infty} \sum_{k=1}^{n}\left(\alpha_{k m} e^{2 m \pi i x / \rho_{k}}+\beta_{k m} e^{-2 m \pi i x / \rho_{k}}\right) .
$$

In what follows in this section we shall suppose that no two of the numbers $\rho_{k}$ have a real ratio. Then no two terms in the series (3.12) involve the same exponential function.

With each of the functions

$$
1, e^{2 m \pi i x / \rho_{k}}, e^{-2 m \pi i x / \rho_{k}} \quad(k=1,2, \cdots, n ; m=1,2,3, \cdots)
$$

let us associate its reciprocal and let us call this associated function the adjoint of the given function. If we multiply any whatever function of the set (3.13) by the adjoint of any other function in the set, we have a product of the form

$$
\prod_{k=1}^{n} e^{2 l_{k} \pi i x / \rho_{k}}
$$

where at least one and not more than two of the integers $l_{k}$ are different from zero. There is a side of the polygon $P$ on which $x / \rho_{k}$ ranges from 0 to 1 ; on that side we denote $x / \rho_{k}$ by $\lambda_{k}$. Then

$$
\int_{0}^{1} \int_{0}^{1} \cdots \int_{0}^{1}\left(\prod_{k=1}^{n} e^{2 l_{k} \pi i \lambda_{k}}\right) d \lambda_{1} d \lambda_{2} \cdots d \lambda_{n}=0
$$

If a like integral is formed with a function of the set (3.13) and the adjoint of that function then the integral corresponding to (3.14) has the value 1. Hence we have conditions of biorthogonality generalizing those pertaining to the case of Fourier series, here arising when $n=1$. Consequently we have a formal method of determining the coefficients in series (3.12) for a much more extensive class of functions than those for which we have already established the validity of such expansions. This suggests the generalization of the whole

* Series similar to those in (3.12) have been treated by P. Bohl, Magisterdissertation, Dorpat, 1893, and Journal für Mathematik, vol. 131 (1906), pp. 286-321. 
theory of Fourier series to the particular class of series in (3.12) if not indeed to the more general class of $\$ 3.3$; but we shall not now pursue these generalizations.

Other generalizations of the whole theory developed in this part of the paper will readily occur to the reader, including among others such extensions of the Birkhoff expansion theory as are parallel to the foregoing extension of the theory of Fourier series and also the extensions of these theories to the expansions of functions of several variables; but these also we leave to a future investigation.

3.5. Applications to Bernoulli polynomials. The theory in $\$ 3.4$ affords elegant expansions of Bernoulli polynomials of higher order, namely, the polynomials $B$ defined by the identity

$$
\frac{\rho_{1} \rho_{2} \cdots \rho_{n} t^{n} e^{x t}}{\left(e^{\rho_{1} t}-1\right) \cdots\left(e^{\rho_{n} t}-1\right)}=\sum_{\nu=0}^{\infty} \frac{t^{\nu}}{\nu !} B_{\nu}^{(n)}\left(x \mid \rho_{1}, \cdots, \rho_{n}\right) .
$$

From this identity we have

$$
B_{\nu}^{(n)}\left(x \mid \rho_{1}, \cdots, \rho_{n}\right)=\frac{\nu ! \rho_{1} \rho_{2} \cdots \rho_{n}}{2 \pi i} \int_{C} \frac{t^{\nu} e^{x t}}{\left(e^{\rho_{1} t}-1\right) \cdots\left(e^{\rho_{n} t}-1\right)} \frac{d t}{t^{\nu+1}}
$$

where $C$ denotes a small circle about the point zero. Our theory is effective for values of $\nu$ not less than $n$.

Thus we have in particular the expansion

$$
\begin{aligned}
B_{\nu}^{(2)}(x \mid 1, i)= & \frac{\nu !}{(2 \pi)^{\nu-1}} \sum_{k=1}^{\infty} \frac{1}{k^{\nu-1}}\left\{\frac{e^{2 k \pi x}}{e^{2 k \pi}-1}+\frac{e^{-2 k \pi x}}{e^{-2 k \pi}-1}+(-i)^{\nu-2} \frac{e^{2 k \pi i x}}{e^{-2 k \pi}-1}\right. \\
& \left.+(-i)^{\nu-2} \frac{e^{-2 k \pi i x}}{e^{2 k \pi}-1}\right\} \quad(\nu=3,4,5, \cdots) .
\end{aligned}
$$

According to the general theory this series must converge for those values $x, x=u+i v$, for which $u$ and $v$ run independently over the closed interval (01). By considering separately the four cases $u<0, u>1, v<0, v>1$, it is easily shown that the series diverges in each case through having terms in the brackets become infinite in an exponential way as $k$ becomes infinite. Hence the whole region of convergence of the series is the square whose vertices are $0,1,1+i, i$.

From this example it follows that the polygon $P$ of convergence in the case of the general theory can not be extended to a larger region in which the series always converges.

UNIVERSITY OF ILLINOIS, URBANA, ILL. 